\title{
LOW-LYING DIRAC EIGENMODES, TOPOLOGICAL CHARGE FLUCTUATIONS AND THE INSTANTON LIQUID MODEL
}

\author{
I. HORVÁTH, S.J. DONG, T. DRAPER \\ Department of Physics \&s Astronomy \\ University of Kentucky, Lexington, KY 40503, USA \\ F.X. LEE \\ Center for Nuclear Studies and Department of Physics \\ George Washington University \\ Washington, DC 20052, USA, and \\ Jefferson Laboratory, Newport News, VA 23606, USA \\ H.B. THACKER \\ Department of Physics \\ University of Virginia, Charlottesville, VA 22901, USA \\ AND \\ J.B. ZHANG \\ CSSM and Department of Physics and Mathematical Physics \\ University of Adelaide, Adelaide, SA 5005, Australia
}

\begin{abstract}
The local structure of low-lying eigenmodes of the overlap Dirac operator is studied. It is found that these modes cannot be described as linear combinations of 't Hooft "would-be" zeromodes associated with instanton excitations that underly the Instanton Liquid Model. This implies that the instanton liquid scenario for spontaneous chiral symmetry breaking in QCD is not accurate. More generally, our data suggests that the vacuum fluctuations of topological charge are not effectively dominated by localized lumps of unit charge with which the topological "would-be" zeromodes could be associated.
\end{abstract}

Presented by I. Horváth at the NATO Advanced Research Workshop "Confinement, Topology, and other Non-Perturbative Aspects of QCD", January 21-27, 2002, Stará Lesná (Slovakia). 


\section{Introduction}

The idea of the instanton-dominated QCD vacuum appeared shortly after the discovery of the instanton [1]. In particular, the instanton gas picture [2] populates the vacuum with well-separated instantons which, using semiclassical methods, leads to the possible qualitative resolution of the $U(1)$ problem [3], as well as to the conclusion that QCD physics depends on the $\theta$-parameter [4]. However, it became clear very soon that QCD is not semiclassical in this sense because the instanton gas picture does not lead to confinement. In fact, it has been argued by Witten [5] that large quantum fluctuations entirely destroy the semiclassical vacuum and that individual instantons do not play a significant dynamical role in QCD.

The fundamental importance of instantons thus became clearly questionable, but the instanton solution served as a basis for a phenomenological Instanton Liquid Model (ILM) [6]. While there are correlations among ILM instantons, the ILM vacuum is still a dilute medium where the instantons of size $\rho \approx 1 / 3 \mathrm{fm}$ and density $n=1 \mathrm{fm}^{-4}$ preserve their identity. It is thus possible to associate a 't Hooft "would-be" zeromode with an individual instanton. The mixing of these modes and a formation of "topological subspace" of low-lying modes is a basis for an elegant mixing scenario for spontaneous chiral symmetry breaking $(\mathrm{S} \chi \mathrm{SB})[]]$. Our goal will be to examine whether this effective picture can be recognized in the low-lying Dirac

modes and thus whether one should assign a fundamental significance to it. The main conclusion from this study is that this is not the case [8] and that the ILM picture is not microscopically accurate.

Our approach is entirely based on studying the local properties of the fermionic eigenmodes [8, 9]. This is entirely appropriate if one's goal is to study the effects of topological charge fluctuations on $\mathrm{S} \chi \mathrm{SB}$. The underlying rough gauge fields are not manipulated in any way, while the fermionic dynamics generates the appropriately smooth behavior automatically. Consequently, this represents an unbiased approach to study the possible dynamical relevance of ILM instantons, as well as the applicability of the topological mixing scenario in general.

The nature of the questions we ask can be described by starting from the ILM picture of the vacuum, or from any picture with vacuum populated by individual instantons preserving their identity. In a typical configuration, there is a collection of smooth (anti)self-dual potential wells for (right)lefthanded components of the fermion. In Fig. 1(A) we show schematically the section of the instanton potential in a direction of another instanton. The potential is truly instanton-like around the core with modifications close to edges due to interactions. The corresponding left-handed 't Hooft modes are shown as well. In equilibrium QCD configuration there are certainly 

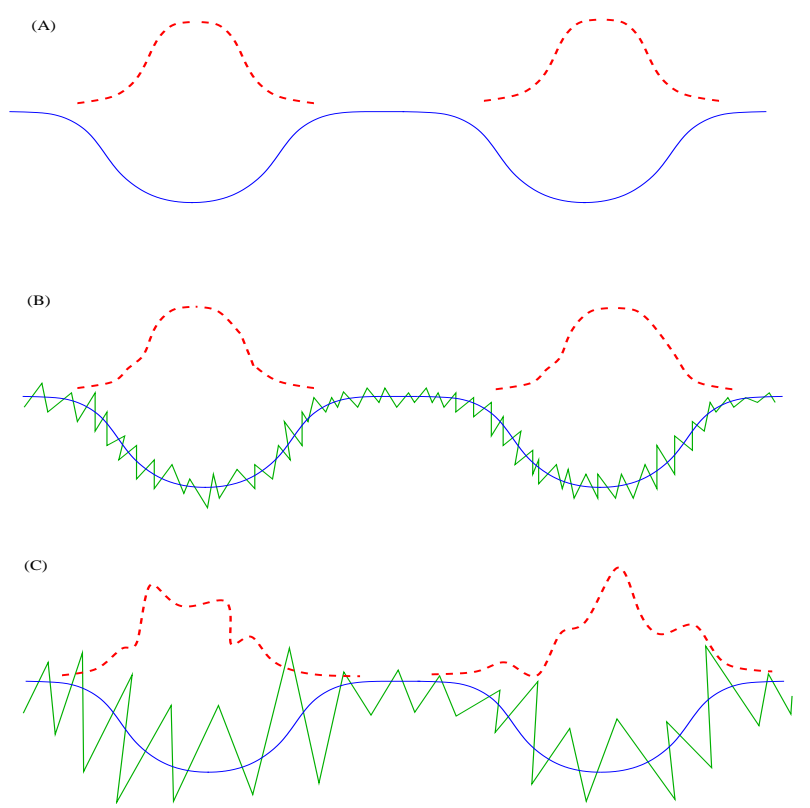

Figure 1. Short-distance fluctuations (rough line) imposed on the instanton-like gauge potential (smooth solid line) and its possible effects on 't Hooft modes (dashed line). See discussion in the text.

additional short-distance fluctuations present. However, these do not necessarily affect the low-momentum propagation of light quarks significantly. Indeed, the infrared Dirac modes filter out the ultraviolet fluctuations that are not important for low-energy fermion dynamics [8]. It would thus be interesting to determine which of the following physically distinct possibilities takes place: (I) The strength and nature of quantum fluctuations is such that the structure of t' Hooft "would-be" zeromodes can still be identified in the true eigenmodes as represented in Fig. 1(B). In this case the ILM scenario for S $\chi \mathrm{SB}$ could indeed be microscopically accurate. (II) Quantum fluctuations deform the 't Hooft modes to the extent that their original structure can not be recognized anymore (see Fig. 1(C)), but the unit quantization of topological charge still takes place in the QCD vacuum. In this case the ILM scenario would not be accurate and it is questionable whether the underlying gauge structures should be referred to as "instantons". However, as pointed out in Ref. [8], the subspace of topological modes would nevertheless be created and thus the basic mechanism proposed in the context of ILM would be operating. (III) Quantum fluctuations are so strong that they destroy unit lumps. In this case a new microscopic origin of Dirac near-zeromodes needs to be sought.

We will present data which rules out option (I) and strongly suggests 


\begin{tabular}{|c|c|c|c|}
\hline$\beta$ & $a[\mathrm{fm}]$ & $V$ & \# configs \\
\hline 5.85 & 0.123 & $10^{3} \times 20$ & 12 \\
\hline 6.00 & 0.093 & $14^{4}$ & 12 \\
\hline 6.20 & 0.068 & $20^{4}$ & 8 \\
\hline 6.55 & 0.042 & $32^{4}$ & 5 \\
\hline
\end{tabular}

TABLE 1. Ensembles of Wilson gauge configurations.

that (II) is not applicable either. Since this talk has been given, the possibility (II) has been excluded directly [10], leaving us with option (III).

\section{Structures in the Eigenmodes of the Overlap Operator}

The microscopic viability of the ILM picture for S $\chi \mathrm{SB}$ can in principle be verified on the lattice by inspecting the local structure of low-lying Dirac modes [9]. In the absence of exact chiral symmetry this should be approached with some care, but since chirally symmetric fermionic actions are now available [11], such strategy is very appropriate. We have calculated low-lying eigenmodes of the overlap Dirac operator [11] in Wilson gauge backgrounds over a large span of lattice spacings as summarized in Table 1 . The physical volumes are chosen to contain on average 3-4 instantons and antiinstantons if the ILM scenario is relevant, ensuring that the mixing of 't Hooft modes would take place, and that "topological subspace" of low-lying modes would form. If that happened, then the local structure of these modes would have very specific local properties related to the structure of underlying 't Hooft modes themselves. The logic of our approach is to assume that this is indeed the case, and to verify the consistency of such assumption against the true local behavior of low-lying modes. To do that, we identify the "structures" in the low-lying modes in such a way that (a) they would correspond to individual 't Hooft modes if ILM scenario was accurate and that (b) they can not arise accidentally as an artifact of the chosen procedure.

As a first step toward defining individual structures correspondingly, we study the $X$-distributions in lowest-lying near-zeromodes [9]. For a given eigenmode $\psi(n)$ the local chiral orientation parameter $X(n)$ is defined as

$$
\tan \left(\frac{\pi}{4}(1+X)\right)=\frac{\left|\psi_{L}\right|}{\left|\psi_{R}\right|}
$$

where $\psi_{L}, \psi_{R}$ are the left and right spinorial components. $X(n)$ represents an angle in the $\left|\psi_{L}\right|-\left|\psi_{R}\right|$ plane rescaled so that $X(n)=-1$ for a purely 

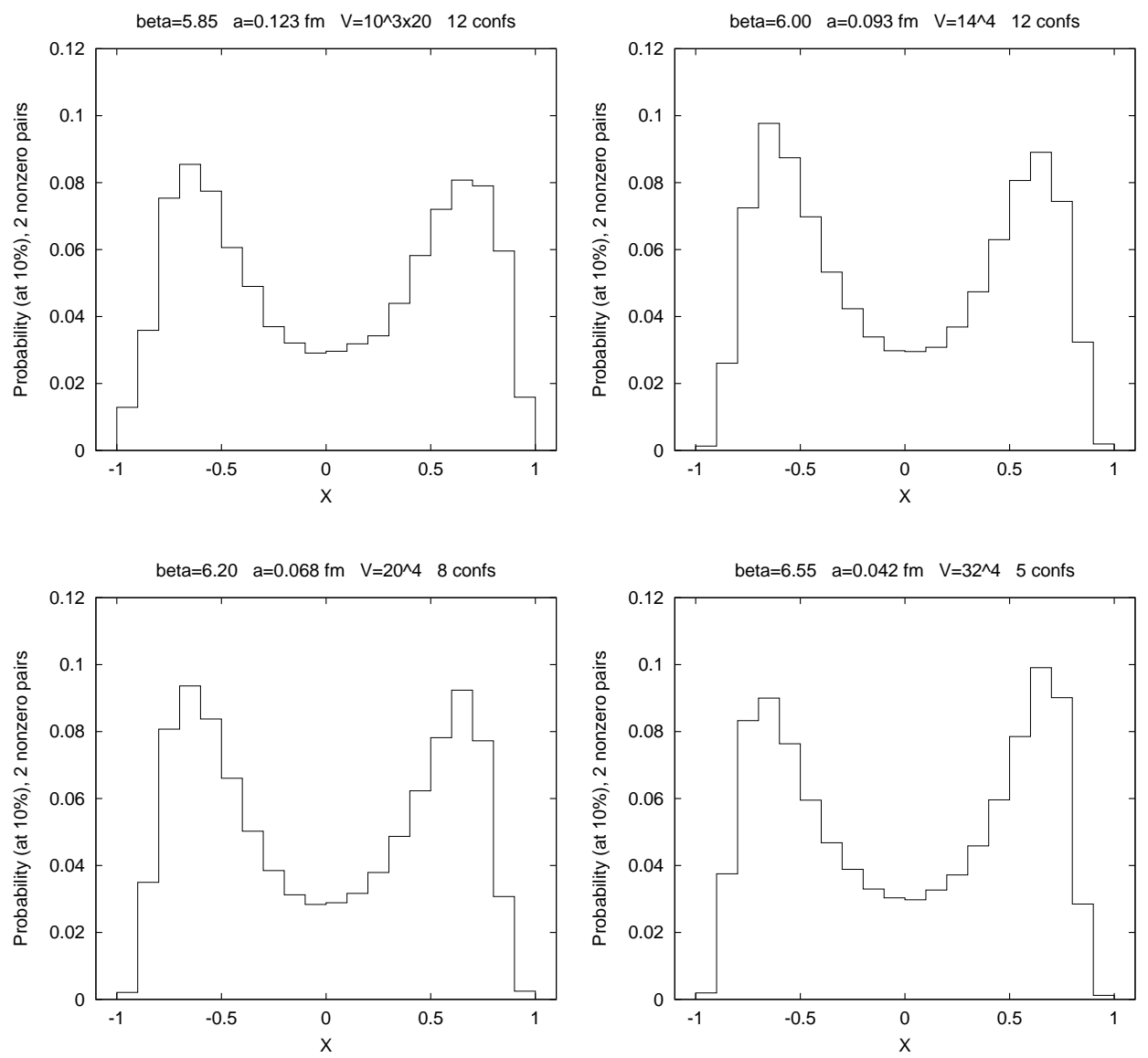

Figure 2. $X$-distributions for four Wilson gauge ensembles considered.

right-handed spinor and $X(n)=+1$ for a purely left-handed one. For modes in the topological subspace the probability distribution of $X$ over the subvolumes occupied by (anti)instantons should be strongly peaked around \pm 1 . On the other hand, if in the subvolume with strong fields the corresponding spinors were strictly unpolarized, a uniform distribution would result.

We have calculated the lowest two pairs of near-zeromodes for the ensembles in Table 1 . The corresponding $X$-distributions shown in Fig. 2 were obtained by considering the fraction $f=1 / 10$ of the volume, where the density $d(n)=\psi_{n}^{+} \psi_{n}$ is highest. This is plausible since the ILM estimates of the instanton packing fraction range between $f \approx 1 / 20$ to $f \approx 1 / 8$. Also, the results are quite insensitive to variations of $f$ in the above range. The 
$X$-distributions scale well, and a double-peaked behavior with maxima at $X \approx \pm 0.65$ emerges (see also Refs. [12]). As Fig. 2 shows, we do not obtain convincing peaks at around \pm 1 as one would naively expect from ILM (The calculated distributions for ILM ensembles are not available in the literature.). However, we use these $X$-distributions as a starting point to verify whether the double-peak behavior is due to the existence of local structures resembling the ILM instantons.

Following the above considerations we identify the local "structure" in the eigenmode by specifying the position $n$ of a local maximum of density $d(m)$ over the distance $\sqrt{3}$, such that (i) $|X(n)| \geq 0.5$, (ii) $n$ belongs to the subvolume used for calculating the $X$-distribution, and (iii) the density decays on average over the distance $\sqrt{3}$ in eight basic lattice directions [8]. This definition satisfies the criteria (a),(b) discussed above and the resulting structures contribute to the maxima of the $X$-distribution by construction. To see whether a structure positioned at $n$ resembles the 't Hooft mode, we have attempted to fit the average profile of the density $d_{n}(r) \equiv<d(m)>_{|n-m|=r}$ with the 't Hooft profile. The situation for a typical structure is shown in Fig. 3. As can be seen, the fits over different regions are very poor and inconsistent with one another. We were simply unable to identify space-time structures whose origin could be directly traced to 't Hooft zeromodes.

\section{Sizes and Densities of the Structures}

As a next step we wish to establish whether the density and the size of structures in the eigenmodes are compatible with the ILM. In Fig. 4 we show the lattice-spacing dependence of density indicating strikingly larger values than assumed in the ILM. There is a rapid growth at small lattice spacings and we can not exclude the potential divergence in the continuum limit. This implies that the true local behavior of topological charge density filtered through infrared eigenmodes is much richer than the one pictured in the ILM.

From our discussion in the previous section it follows that the sizes of individual structures can not be determined from fits to the 't Hooft profile (see Ref. [8] for details). We thus adopt a different procedure based only on the assumption of topological mixing. In particular, the "wouldbe" zeromodes localized on the unit quantized gauge lumps would produce regions of coherent local chirality of some typical size. It is thus natural to assign the radius $R_{n}$ to the structure at $n$ as the radius of the largest hypersphere centered at $n$, containing the points with the same sign of local chirality $\psi_{m}^{+} \gamma_{5} \psi_{m}$. We have determined the average radius for our ensembles and the results are shown in Fig. 1 . The data shows that this 


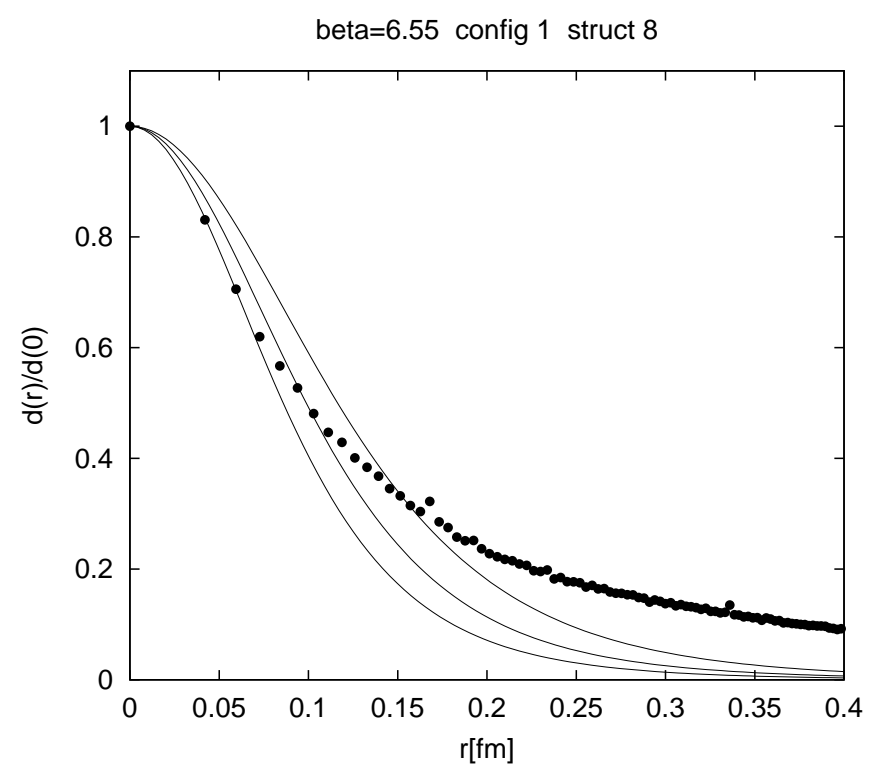

Figure 3. The profile of the typical structure and attempted fits to 't Hooft profile in the region $0.00-0.06 \mathrm{fm}$ (leftmost curve), $0.06-0.12 \mathrm{fm}$ (middle curve), and $0.12-0.18 \mathrm{fm}$ (rightmost curve).

quantity has a finite continuum limit characterizing the typical size of the coherent regions. The corresponding scale we obtain in the continuum limit is much smaller than the one considered in the ILM.

\section{The Consequences of the Numerical Results}

The results from Sections 2 and 3 clearly demonstrate that the local structure of Dirac near-zeromodes for equilibrium QCD backgrounds differs significantly from the behavior associated with the ILM picture of the vacuum. This conclusion does not relate only to the obvious quantitative disagreement with parameters of the ILM that we observe. Indeed, the fact that we are not able to identify structures with 't Hooft profile indicates that a generic picture where vacuum is populated by relatively independent instantons preserving their individual identity is not microscopically accurate. We have thus excluded the possibility (I) of the Introduction.

We now consider the merit of the possibility (II), namely that quantum fluctuations, while deforming the ILM instantons severely, do not destroy the integrity of unit topological charges. In such vacuum there would be identifiable individual lumps of unit topological charge present and the topological mixing scenario of $\mathrm{S} \chi \mathrm{SB}$ would still be relevant [8]. If $\chi^{j}$ is a 

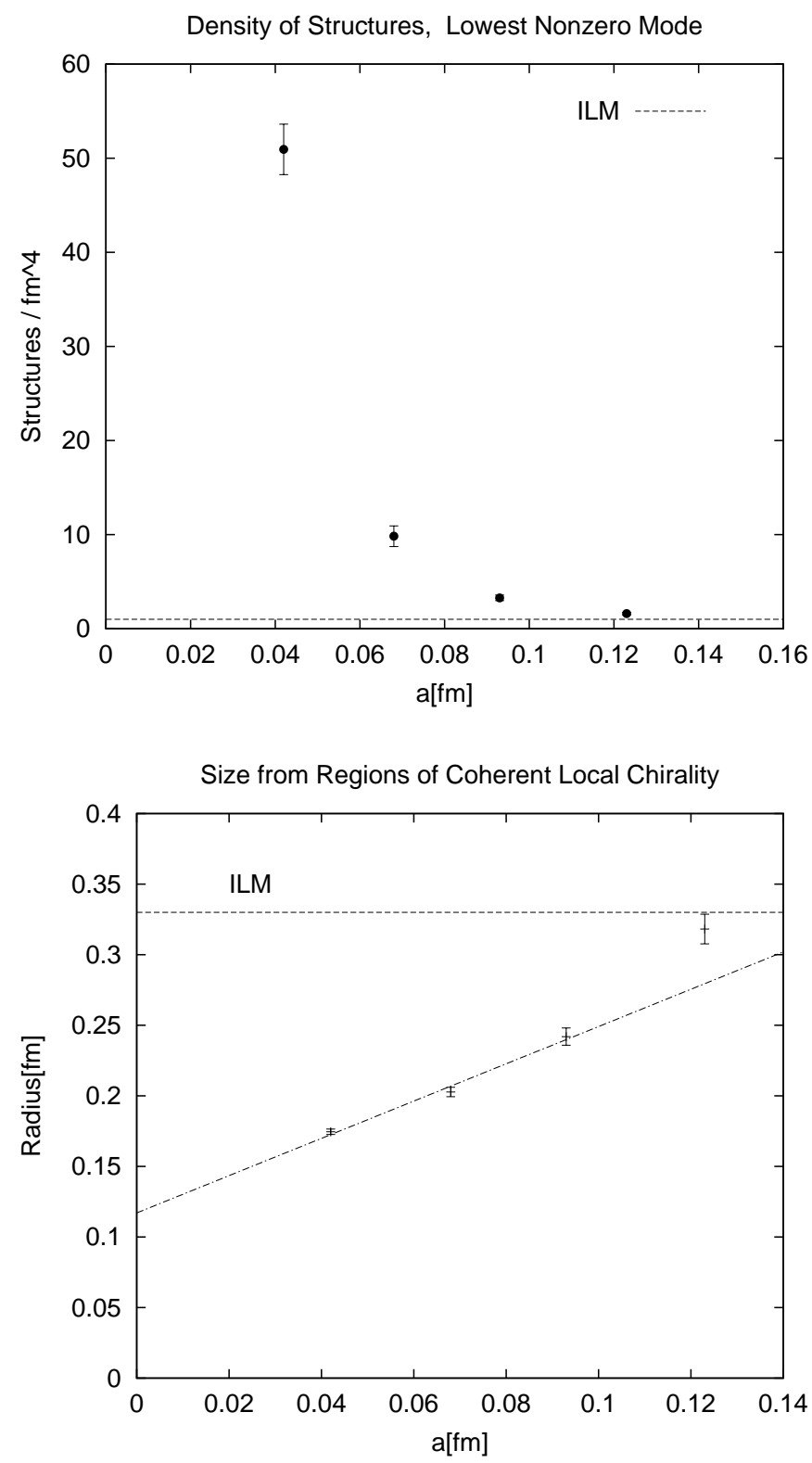

Figure 4. Top: Density of structures (in $\mathrm{fm}^{-4}$ ) as a function of the lattice spacing. Bottom: Average radius, $\left\langle R_{n}\right\rangle$, of structures from regions of coherent local chirality. The lowest nonzero mode was used for the calculation. Data for the three smallest lattice spacings were used to obtain the fit. The horizontal line represents the radius of an ILM instanton. 


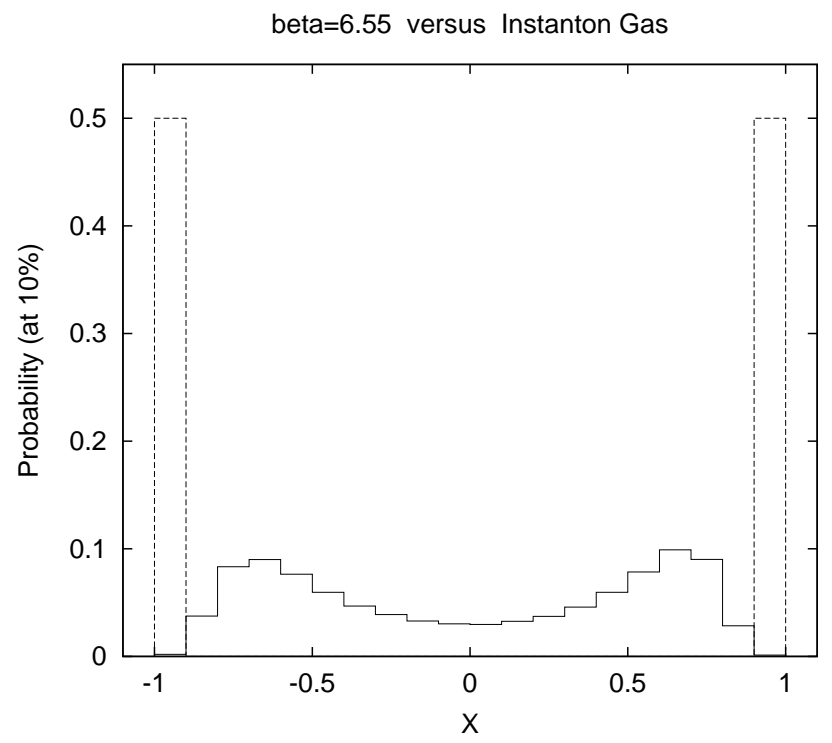

Figure 5. Comparison of $X$-distributions at $\beta=6.55$ for near-zero modes (solid line) and exact zero modes (dashed line) using the overlap Dirac operator.

chiral "would-be" zeromode associated with a given unit lump, then the topological subspace of modes $\psi^{i} \approx \sum_{j} a_{i j} \chi^{j}, i=1, \ldots N_{L}$ would form if $N_{L}$ lumps were present in the volume. Even for general unit lumps this situation implies very specific properties that can be tested.

First, if a topological subspace is formed, then all eigenmodes in the subspace should have very similar local properties since they are all the descendants of the chiral "would-be" zeromodes localized on the lumps. As a consequence, there should be no qualitative difference in this regard between the zeromodes and the near-zero modes belonging to the subspace [9]. The $X$-distribution is an ideal tool for testing whether this is the case or not. In Fig. 5 we compare the $X$-distribution for two pairs of nearzeromodes from our ensemble at $\beta=6.55$ (our finest lattice spacing) to the $X$-distribution for the zeromodes [8]. Instead of qualitative agreement, we clearly observe a very abrupt change in the behavior of the near-zeromodes.

At the same time, the topological mixing scenario predicts that there should be a sudden qualitative change in the local behavior at the point in the spectrum where the topological subspace ends. In particular, the $X$-distribution should be much less peaked (or not peaked at all) for eigenmodes outside the topological subspace. The number of unit topological lumps $N_{L}$ (i.e. the dimension of the topological subspace) can be estimated 

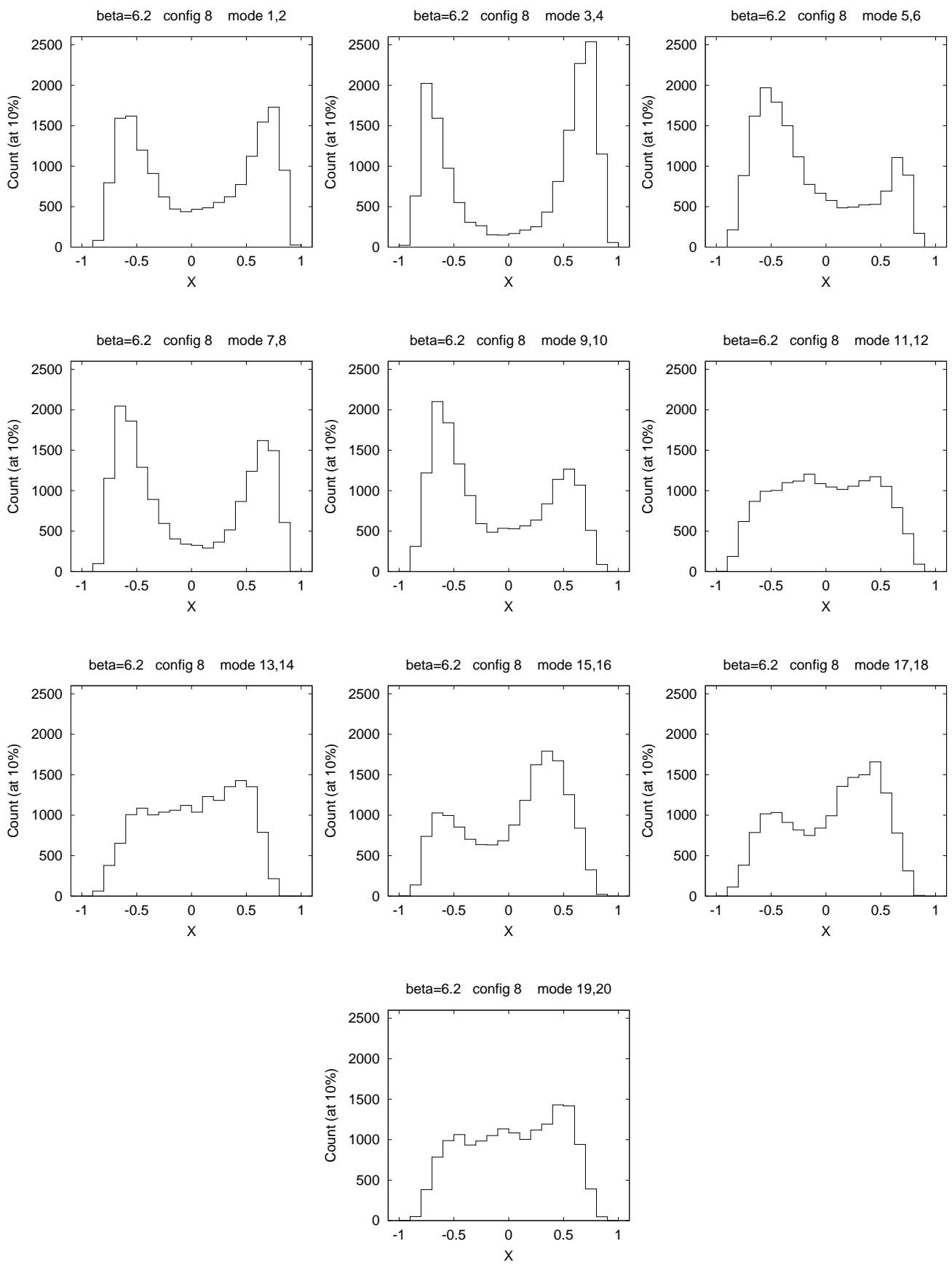

Figure 6. The $X$-distributions for first 20 near-zero modes of configuration 8 at $\beta=6.2$. 
from the known value of pure gauge topological susceptibility $\left(\approx 1 \mathrm{fm}^{-4}\right)$. Since the lumps enter as individual entities, they are relatively independent, and one should observe $N_{L} \approx V \approx 3-4$ for our ensembles. For several configurations we have calculated up to 20 near-zeromodes to make sure that the dimension of the topological subspace could be identified. In Fig. 6 we illustrate the typical behavior by displaying results for configuration 8 from our $\beta=6.2$ ensemble. This configuration has $Q=0$ and we show the $X$-histograms for 10 pairs of near-zeromodes (the histogram is the same for both modes in a pair). Inspecting these results reveals that there are at least 14 modes with similar double-peaked structure instead of 3-4. Moreover, the decrease in double-peaking appears to be gradual and there is every reason to expect that higher modes will be peaked as well.

The above considerations show quite clearly that the idea of the distinctive topological subspace being the source of the low-lying eigenmodes generating the finite average microscopic density around zero (and hence $\mathrm{S} \chi \mathrm{SB}$ [13]) is not consistent with our data. This suggest that the logical possibility (II) described in the Introduction is probably not what happens in the true vacuum of pure gauge QCD.

\section{Conclusions}

In this work we have demonstrated that the ILM scenario for $\mathrm{S} \chi \mathrm{SB}$ is not microscopically accurate. In particular, the low-lying Dirac modes cannot be described as mixtures of 't Hooft modes associated with ILM instantons. More generally, our data suggests that the bulk of topological charge in QCD is not effectively concentrated in quantized unit lumps to which it would be possible to assign the corresponding "would-be" zeromodes. Since this talk has been given, this suggestion has been put on a firm ground and demonstrated directly in Ref. [10]. Our findings imply that a qualitatively different mechanism for the origin of the Dirac near-zeromodes should be sought.

\section{Acknowledgment}

IH thanks the organizers of the workshop for a very well-organized, informative, and exceptionally pleasant meeting. He also acknowledges an interesting conversation with H. Reinhardt and M. Engelhardt.

\section{References}

1. Belavin, A.A., Polyakov, A.M., Schwartz, A., Tyupkin, Y. (1975) Pseudoparticle solutions of the Yang-Mills equations, Physics Letters B59, 85

2. Callan, C.G., Dashen, R., Gross, D.J. (1978) Toward a theory of the strong interactions, Physical Review D17, 2717 
3. 't Hooft, G. (1976) Symmetry breaking through Bell-Jackiw anomalies, Physical Review Letters 37, 8; 't Hooft, G. (1976) Computation of the quantum effects due to a four-dimensional pseudoparticle, Physical Review D14, 3432

4. Callan, C.G., Dashen, R., Gross, D.J. (1976) The structure of the gauge theory vacuum, Physics Letters B63, 334; Jackiw, R., Rebbi, C. (1976) Vacuum periodicity in a Yang-Mills quantum theory, Physical Review Letters 37, 172

5. Witten, E. (1979) Instantons, the quark model, and the $1 / \mathrm{N}$ expansion, Nuclear Physics B149, 285

6. Shuryak, E.V. (1982) Hadrons containing a heavy quark and QCD sum rules, Nuclear Physics B198, 83; Diakonov, D.I., Petrov, V.Y. (1984) Instanton based vacuum from Feynman variational principle, Nuclear Physics B245, 259; Schäfer, T., Shuryak, E. (1998) Instantons in QCD, Reviews of Modern Physics 70, 323

7. Diakonov, D.I., Petrov, V.Y. (1986) A theory of light quarks in the instanton vacuum, Nuclear Physics B272, 457

8. Horváth, I., et al. (2002) Local chirality of low-lying Dirac eigenmodes and the Instanton Liquid model, hep-lat/0201008; Dong, S.J., et al. (2002) Topological charge fluctuations and low-lying Dirac eigenmodes, Nuclear Physics (Proc. Suppl.) 106, 563

9. Horváth, I., Isgur, N., McCune, J., Thacker, H.B. (2002) Evidence against instanton dominance of topological charge fluctuations in QCD, Physical Review D65, 014502

10. Horváth, I.. et al. (2002) On the local structure of topological charge fluctuations in QCD, hep-lat/0203027

11. Neuberger, H. (1998) Exactly massless quarks on the lattice, Physics Letters B417, 141; Neuberger, H. (1998) More about exactly massless quarks on the lattice, Physics Letters B427, 353

12. DeGrand, T., Hasenfratz, A. (2002) Comment on "Evidence Against Instanton Dominance of Topological Charge Fluctuations in QCD", Physical Review D65, 014503; Hip, I. et al. (2002) Instanton dominance of topological charge fluctuations in QCD?, Physical Review D65, 014506; Edwards, R., Heller, U. (2002) Are topological charge fluctuations in QCD instanton dominated?, Physical Review D65, 014505; Blum, T., et al. (2002) Chirality Correlation within Dirac eigenvectors from Domain Wall Fermions, Physical Review D65, 014504; Gattringer, C., et al. (2001) A comprehensive picture of topological excitations in finite temperature lattice QCD, Nuclear Physics B618, 205

13. Banks, T., Casher, A. (1980) Chiral symmetry breaking in confining theories, Nuclear Physics B169, 103 